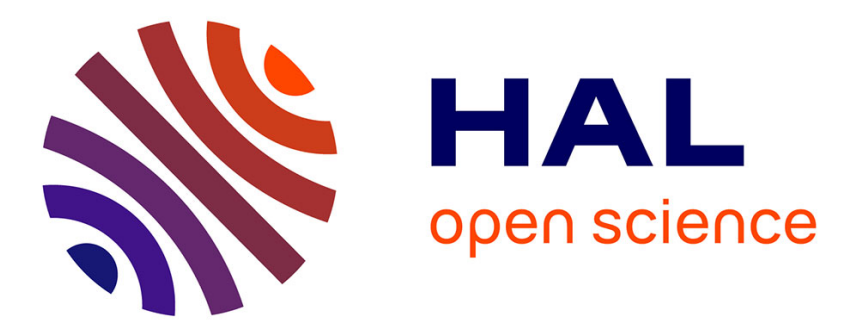

\title{
Test of single degree of freedom acoustic treatment impedance models for multimodal acoustic propagation in duct with flow
}

Ryan Baccouche, Solène Moreau, Mabrouk Ben Tahar

\section{- To cite this version:}

Ryan Baccouche, Solène Moreau, Mabrouk Ben Tahar. Test of single degree of freedom acoustic treatment impedance models for multimodal acoustic propagation in duct with flow. Journal of the Acoustical Society of America, 2017, 141 (6), pp.4168-4178. 10.1121/1.4983653 . hal-02180353

\section{HAL Id: hal-02180353 \\ https://hal.utc.fr/hal-02180353}

Submitted on 11 Jul 2019

HAL is a multi-disciplinary open access archive for the deposit and dissemination of scientific research documents, whether they are published or not. The documents may come from teaching and research institutions in France or abroad, or from public or private research centers.
L'archive ouverte pluridisciplinaire HAL, est destinée au dépôt et à la diffusion de documents scientifiques de niveau recherche, publiés ou non, émanant des établissements d'enseignement et de recherche français ou étrangers, des laboratoires publics ou privés. 
Test of single degree of freedom acoustic treatment impedance models for multimodal acoustic propagation in duct with flow

Ryan Baccouche, Soléne Moreau, and Mabrouk Ben Tahar

Citation: The Journal of the Acoustical Society of America 141, 4168 (2017); doi: 10.1121/1.4983653

View online: http://dx.doi.org/10.1121/1.4983653

View Table of Contents: http://asa.scitation.org/toc/jas/141/6

Published by the Acoustical Society of America 


\title{
Test of single degree of freedom acoustic treatment impedance models for multimodal acoustic propagation in duct with flow
}

\author{
Ryan Baccouche, Soléne Moreau, ${ }^{\text {a) }}$ and Mabrouk Ben Tahar \\ Sorbonne University, Université de Technologie de Compiègne, CNRS, Unité Mixte de Recherche 7337 \\ Roberval, Centre de Recherche Royallieu, CS 60319, 60203 Compiègne cedex, France
}

(Received 8 November 2016; revised 30 March 2017; accepted 2 May 2017; published online 6 June 2017)

\begin{abstract}
Passive acoustic treatments, also called liners, are the main solution to noise problems. The Single Degree Of Freedom (SDOF) acoustic treatment, composed of a thin material (perforated plate) affixed to air cavities with a rigid bottom, constitutes a solution. Predicting sound level reduction by an SDOF treatment requires reliable acoustic impedance models. An experimental/numerical method has been developed for a duct with an acoustic treatment to test acoustic impedance models of SDOF treatment with a multimodal propagation in the presence of a mean flow. This method is based on the comparison of experimental results from an aeroacoustic bench composed of a circular duct with a treated area, and numerical results from an FEM-PML axisymmetric model based on Galbrun's equation. The numerical results are confronted with experimental results to test impedance models up to $M_{0}= \pm 0.25$. (C) 2017 Acoustical Society of America.
\end{abstract}

[http://dx.doi.org/10.1121/1.4983653]

$[\mathrm{RR}]$

Pages: $4168-4178$

\section{INTRODUCTION}

With regulations becoming increasingly strict about noise levels in the transport sector, it has become crucial for manufacturers to make an effort to reduce the noise of their products. This is the case in aeronautics, where airport requirements are increasingly drastic for aircraft.

Passive acoustic treatments, also called liners, are the main solution to the noise reduction problems for this application. They must meet several requirements, especially for aircraft turbojet engines, ${ }^{1}$ where conditions are extreme. In this configuration, the flow can reach Mach number of 0.3 (take-off and landing phase). The acoustic treatment that best meets these requirements is the Single Degree Of Freedom (SDOF) treatment. It consists of a thin material affixed to air cavities with a rigid bottom (see Fig. 1). The thin material, or resistive layer, is composed of a perforated plate on which a wire mesh can be bonded. The liner's acoustic properties are characterized by its impedance, which depends on its geometric structure and operating conditions. In the literature, semi-empirical models characterizing the SDOF treatment impedance are widespread. Some of these models take into account the influence of the mean flow. These models are generally based on a mixed analyticalempirical approach. However, their validation is often performed on flat plates and a normal incidence wave excitation. In this paper, we will estimate the validity of these models in a cylindrical configuration with multimodal excitation and mean flow can reach a Mach number $M_{0}= \pm 0.25$.

The organization of this paper is as follows: In Sec. II, some of the acoustic impedance models are presented. Section III is intended to introduce the underlying theory and variational formulation of the aeroacoustic Galbrun equation. The

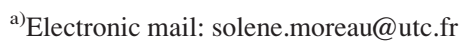

numerical model is based on an axisymmetric finite element model. In Sec. IV, the experimental and data processing steps are presented. In Sec. V, two SDOF treatments are tested: one with a perforated plate (hole diameter $1.9 \mathrm{~mm}$ ) and the other with a microperforated plate (hole diameter $0.3 \mathrm{~mm}$ ). The experimental-numerical correlations for different impedance models submitted to multimodal excitation and different mean flows are presented $\left(M_{0}= \pm 0.25\right)$.

\section{ACOUSTIC IMPEDANCE}

The SDOF treatment is often used for attenuating turbine noise radiating from the jet engines of aircraft. Several semi-empirical models have been proposed to predict their acoustic impedance. Whereas viscous, radiation and backing effects result from semi-analytical solutions, other effects are often based on an empirical approach. Much effort has been made to correct terms, especially when a grazing flow or high sound pressure level is present. These terms can easily under- or over-predict the impedance, as a consequence of the assumptions made. We present in the following paragraph some SDOF models from the literature.

\section{A. SDOF acoustic impedance}

The geometric parameters of the SDOF treatments have a great influence on their performance, as well as the frequency range over which they are effective. The SDOF treatment (see Figs. 1 and 2) consists of the following:

- A resistive layer that is composed of a perforated plate whose thickness is much smaller than the wavelength. The parameters of the resistive layer are plate thickness $t$, hole diameter $d$, perforation rate $\sigma=n(s / S)$ with $n$ the number of holes in the plate, $s$ one hole area and $S$ total area.

- Air cavities of small diameter compared to the wavelength. The bottom of the cavity $(y=0)$ is generally rigid. 


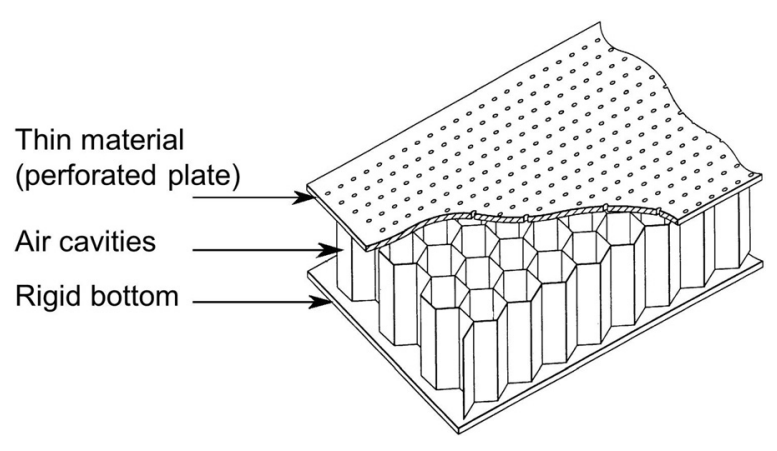

FIG. 1. Sketch illustrating SDOF acoustic treatment.

Under the assumption of a local reaction, the acoustic incident wave can propagate in the cavity in only one direction (flowing along the $y$ axis). In addition, the wave number $k=\omega / c_{0}$ ( $\omega$ is the angular frequency and $c_{0}$ the speed of sound) in the cavity is the same as the wave propagating in the duct, regardless of its incidence angle. The parameters of the air cavity are the depth of the cavity $L_{c a v}$ and its section $s_{c a v}$.

The total surface impedance of an SDOF treatment (resistive layer and cavities) is defined by

$$
z_{t}=z_{r}+z_{c a v}
$$

with $z_{r}$ the resistive layer impedance and $z_{c a v}$ the cavity impedance.

The resistive layer impedance is given by

$$
z_{r}=\frac{p_{2}^{L}-p_{1}^{L}}{\rho_{0} c_{0} v_{n}^{L}},
$$

with $p_{1}^{L}$ and $p_{2}^{L}$ representing the Lagrangian pressure perturbation on the inner and outer surface of the plate, $v_{n}^{L}$ the normal Lagrangian acoustic velocity perturbation at point 1 and $\rho_{0}$ the air density (see Fig. 2).

The cavity impedance is given by

$$
z_{\text {cav }}=\frac{p_{1}^{L}}{\rho_{0} c_{0} v_{n}^{L}}=\frac{p_{c a v}^{L}\left(L_{c a v}\right)}{-\rho_{0} c_{0} v_{c a v}^{L}\left(L_{c a v}\right)},
$$

with $p_{c a v}^{L}$ and $v_{\text {cav }}^{L}$ the pressure and velocity of acoustic Lagrangian perturbation in the cavity. Under the assumption of a local reaction (plane propagation in the cavity), the

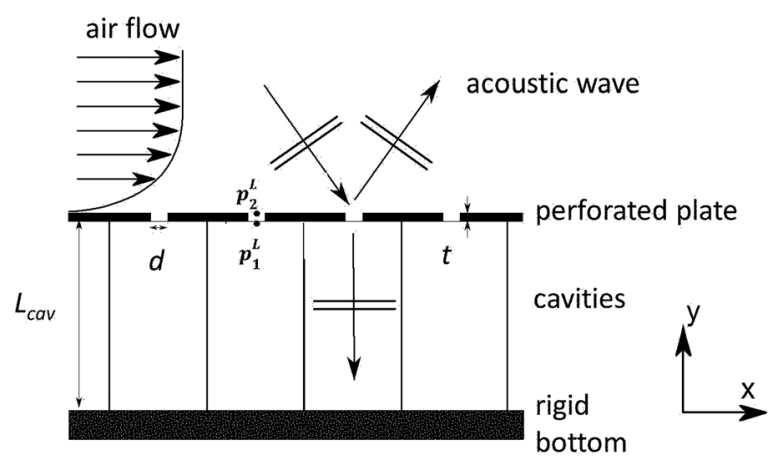

FIG. 2. SDOF treatment with the presence of flow. acoustic pressure is given by $p_{\text {cav }}^{L}(y)=\left(A e^{+i k y}+B e^{-i k y}\right) e^{-i \omega t}$ and velocity by $v_{\text {cav }}^{L}(y)=\left(1 / \rho_{0} c_{0}\right)\left(A e^{+i k y}-B e^{-i k y}\right) e^{-i \omega t}$. The rigid bottom cavity condition gives $v_{\text {cav }}^{L}(0)=0$ and so $A=B$. Thus, the surface impedance of the cavity is given by

$$
z_{c a v}=+i \cot \left(k L_{c a v}\right) \text {. }
$$

The cavity impedance modifies only the reactance term.

Finally, the total impedance of the treatment is given by

$$
z_{t}=z_{r}+i \cot \left(k L_{c a v}\right) .
$$

To determine the impedance $z_{r}$, it is thus necessary to have an impedance model of perforated plates.

\section{B. Acoustic impedance models of perforated plates in the presence of a mean flow}

Some of the semi-empirical models of $z_{r}$ for perforated and microperforated plates are presented in this section. The impedance $z_{r}$ is in general a function of fluid properties $\left(\rho_{0}\right.$, $c_{0}$, and kinematic viscosity $\left.\nu\right)$, liner geometry $(d, t, \sigma$, orifice end corrections $\delta$, resistive layer normalized resistance $\delta_{r}$, and resistive layer normalized reactance $\delta_{\chi}$ ) and operating conditions $\left(\omega\right.$, discharge coefficient $C_{d}$, boundary layer displacement thickness $\delta^{*}$, mean flow velocity in Mach number $M_{0}$, air particle velocity in the orifice in Mach number $M_{a}$ ). The following assumption is often used $t, d, L_{\text {cav }} \ll \lambda$ where $\lambda$ is the wavelength. These models take or not into account the effects of viscosity, acoustic velocity, radiation, mean flow and interaction effects between adjacent holes. The Appendix gives a summary of the effects taken into account by the authors.

\section{Guess' model (1975)}

Guess $^{2}$ provides an acoustic impedance model for perforated plates $(d \geq 1 \mathrm{~mm})$ for low and high frequencies

$$
\begin{aligned}
z_{\mathrm{Guess}, f<100 \mathrm{~Hz}}= & {\left[\frac{32 \nu(t+d)}{\sigma c_{0} d^{2}}+\frac{1}{8 \sigma}(k d)^{2}+\frac{1-\sigma^{2}}{\sigma} M_{a}\right.} \\
+ & \left.K \frac{1-\sigma^{2}}{\sigma} M_{0}\right]-i\left[\frac{4 k t}{3 \sigma}+\frac{k}{\sigma} \delta\right], \\
z_{\mathrm{Guess}, f>1000 \mathrm{~Hz}}= & {\left[\frac{\sqrt{8 \nu \omega}(t+d)}{\sigma c_{0} d}+\frac{1}{8 \sigma}(k d)^{2}\right.} \\
& \left.+\frac{1-\sigma^{2}}{\sigma} M_{a}+K \frac{1-\sigma^{2}}{\sigma} M_{0}\right] \\
& -i\left[\frac{k t}{\sigma}+\frac{\sqrt{8 \nu \omega}(t+d)}{\sigma c_{0} d}+\frac{k}{\sigma} \delta\right],
\end{aligned}
$$

with $K$ an empirical coefficient based on the theory of boundary layers ( $K=0.3$ for perforated plates).

\section{Allam's model (2008)}

The model of Allam and $\AA_{b o m^{3}}$ is based on Bauer's 4 model for microperforated plates in the presence of flow 
$z_{\text {Allam } 2008}=\left[\frac{\sqrt{8 \nu \omega}(t+d)}{\sigma c_{0} d C_{d}}+\frac{K}{\sigma} M_{0}\right]-i\left[\frac{k}{\sigma C_{d}}(t+\delta)\right]$.

\section{Yu's model (2008)}

Yu et al. ${ }^{5}$ propose a semi-empirical impedance model developed for perforated and microperforated plates

$$
\begin{aligned}
z_{Y u}= & {\left[\operatorname{Re}\left(\frac{i k}{\sigma}\left(1-\frac{4 J_{1}\left(k_{s} d / 2\right)}{k_{s} d J_{0}\left(k_{s} d / 2\right)}\right)^{-1}\left(t+d \delta_{r}\right)\right)+\frac{1.336541}{2 C_{d}^{2}} \frac{1-\sigma^{2}}{\sigma^{2}} M_{a}+\frac{1}{\sigma\left(2+1.256 \frac{\delta^{*}}{d}\right)} M_{0}\right] } \\
& -i\left[\operatorname{Im}\left(\frac{i k}{\sigma}\left(1-\frac{4 J_{1}\left(k_{s} d / 2\right)}{k_{s} d J_{0}\left(k_{s} d / 2\right)}\right)^{-1}\left(t+d \delta_{\chi}\right)\right)+S_{m} M_{a}\right]
\end{aligned}
$$

with $k_{s}$ the Stokes number, $J_{0}$ and $J_{1}$ the zero- and first-order Bessel functions of the first kind and $S_{m}$ the nonlinear mass reactance coefficient.

\section{Cobo's model (2010)}

The model of Cobo et al. ${ }^{6}$ is applicable to microperforated plates. It is similar to Allam's ${ }^{3}$ model with changes in the end correction

$$
\begin{aligned}
z_{C o b o}= & {\left[\frac{\sqrt{8 \nu \omega}}{4 c_{0} \sigma}+\frac{K}{\sigma} M_{0}\right] } \\
& -i\left[\frac{k t}{\sigma}\left(1-\frac{4 J_{1}\left(k_{s} d / 2\right)}{k_{s} d J_{0}\left(k_{s} d / 2\right)}\right)^{-1}+\frac{k}{\rho_{0} \sigma} \delta\right] .
\end{aligned}
$$

It was tested on a double perforated plate system combined with a porous medium.

\section{Allam's model (2011)}

To develop a more complete model than the one proposed in 2008, Allam and $\AA^{\circ}$ bom $^{7}$ propose another model. They are inspired by the work of $\mathrm{Maa}^{8-11}$ on microperforated plates

$$
\begin{aligned}
z_{\text {Allam } 2011}= & {\left[\operatorname{Re}\left(\frac{i k t}{\sigma}\left(1-\frac{4 J_{1}\left(k_{s} d / 2\right)}{k_{s} d J_{0}\left(k_{s} d / 2\right)}\right)^{-1}\right)\right.} \\
& \left.\times \frac{\alpha \sqrt{2 \nu \omega}}{\sigma c_{0}}+\frac{1}{\sigma} M_{a}+\frac{K}{\sigma} M_{0}\right] \\
& -i\left[\operatorname{Im}\left(\frac{i k t}{\sigma}\left(1-\frac{4 J_{1}\left(k_{s} d / 2\right)}{k_{s} d J_{0}\left(k_{s} d / 2\right)}\right)^{-1}\right)+\frac{k}{\sigma} \delta\right]
\end{aligned}
$$

with $\alpha$ is a factor for sharp edges.

\section{Experimental validation ranges used by their authors}

Table I summarizes the experimental validation ranges used by the authors.

\section{NUMERICAL MODEL FOR AEROACOUSTIC EQUATION}

For many aeroacoustic applications, the Linearized Euler Equations (LEEs) form the basic starting point for the majority of the currently used Computational AeroAcoustic (CAA) models. ${ }^{12}$ For two decades, we have been developing the Linearised Galbrun Equation (LGE) which can be viewed as an alternative to the LEEs. However, its direct numerical treatment by Finite Element Method (FEM) has been the source of difficulties due to the well-known locking phenomenon, which usually occurs with a purely displacement based formulation. A mixed pressure-displacement FEM formulation has been previously proposed to avoid a spurious solutions phenomenon. ${ }^{13,14}$ This mixed approach is general and easy to implement in FEM code and has been tested successfully for the harmonic/time-domain and bounded/unbounded space domain. ${ }^{15,16}$

\section{A. Mixed variational Galbrun formulation based on pressure-displacement variables}

The propagation of linear acoustic unsteady disturbances in an inviscid non-heat conducting continuous medium can be expressed by a mixed Eulerian-Lagrangian description. This description consists in considering a Lagrangian perturbation of the physical quantities associated to the fluid particles, expressed in terms of geometrical Eulerian variables relatively to a mean steady flow. The linear Lagrangian perturbation is defined by

$$
f^{L}\left(x_{0}, t\right) \equiv f(a, t)-f_{0}(a, t) \equiv f(x, t)-f_{0}\left(x_{0}, t\right),
$$

where $x_{0}$ is the geometrical position of a particle $a$ in the base mean steady flow velocity $\mathbf{v}_{0}$, and $x$ is the geometrical position of the same particle $a$ in the perturbed flow. $f_{0}(a, t)$ is the physical quantity (scalar or tensor) in the base mean flow associated to the particle $a$ and $f(a, t)$ denotes the same quantity in the perturbed flow.

\section{B. The mixed Eulerian-Lagrangian aeroacoustic Galbrun equation}

The application of the mixed description perturbations rules to the Eulerian equations gives the so-called Galbrun equations ${ }^{13,14}$ 
TABLE I. Experimental validation ranges used by their authors.

\begin{tabular}{|c|c|c|c|c|}
\hline Models & Guess & $\mathrm{Yu}$ & Cobo & Allam \\
\hline Plate thickness $t(\mathrm{~mm})$ & 1 & {$[0.8 ; 1.0 ; 1.2 ; 1.3]$} & 0.5 & 1 \\
\hline Hole diameter $d(\mathrm{~mm})$ & 2.5 & {$[1.0 ; 1.1 ; 1.2]$} & {$[0.3 ; 0.5]$} & 1 \\
\hline Perforation rate $\sigma$ & {$[0.20 ; 0.25]$} & {$[0.077 ; 0.079 ; 0.082 ; 0.118]$} & {$[0.23 ; 0.30]$} & {$[0.005 ; 0.02]$} \\
\hline Mach number of mean flow $M_{0}$ & {$[0 ; 0.5]$} & {$[0.2 ; 0.3 ; 0.45 ; 0.5]$} & {$[0 ; 0.1]$} & {$[0 ; 0.15]$} \\
\hline Duct diameter (m) & $?$ & 0.05 (square) & 0.2 (square) & 0.057 (cylinder) \\
\hline Frequency $(\mathrm{Hz})$ & $?$ & {$[500 ; 3500]$} & {$[0 ; 5145]$} & {$[0 ; 1100]$} \\
\hline First mode cutoff frequency $(\mathrm{Hz})$ & $?$ & 3400 & 850 & 3493 \\
\hline
\end{tabular}

$$
\left\{\begin{array}{l}
\rho_{0} \frac{\mathrm{d}_{0}^{2} \mathbf{w}^{L}}{\mathrm{~d} t^{2}}+\nabla p^{L}+\left(\nabla \cdot \mathbf{w}^{L}\right) \nabla p_{0}-{ }^{T} \nabla \mathbf{w}^{L} \cdot \nabla p_{0}=0 \\
p^{L}=-\rho_{0} c_{0}^{2} \nabla \cdot \mathbf{w}^{L}
\end{array}\right.
$$

where $\mathrm{d}_{0} / \mathrm{d} t=\left(-\mathrm{i} \omega+\mathbf{v}_{0} \cdot \nabla\right)$ is the convective derivative, $\mathbf{w}^{L}$ the linear Lagrangian perturbation of the particle displacement vector, $p^{L}$ the linear Lagrangian perturbation of the pressure and $p_{0}$ the mean pressure.

It is assumed that the mean parameters $\left(\rho_{0}, p_{0}, c_{0}, \mathbf{v}_{0}\right)$ verify the Euler equations in a steady isentropic state.

\section{The boundary conditions}

Two types of boundary conditions must be distinguished in the physical domain. The boundary conditions corresponding to the fluid domain in which the displacement or pressure are known (boundary $S_{1}$ ) are directly enforced at nodes as a constraint in the FEM discrete model

$$
\mathbf{w}^{L}=\overline{\mathbf{w}} \quad \text { or } \quad p^{L}=\bar{p} .
$$

The boundary conditions corresponding to the impermeable material walls to the mean flow (boundary $S_{2}$ ) are simply given by the following relation ${ }^{13}$

$$
\mathbf{w}^{L} \cdot \mathbf{n}_{0}=-\frac{1}{\mathrm{i} \omega Z} p^{L}
$$

where $Z$ is the wall impedance of the locally reacting liners $\left(Z \rightarrow \infty\right.$ for a rigid wall) and $\mathbf{n}_{0}$ the wall inward normal vector (outward for the fluid).

When the problems are posed on large or unbounded domains (e.g., an anechoic chamber), numerical methods need efficient and easy to implement techniques to truncate the computational domain without disturbing the solution of the original problem. Several efficient methods have been developed in the literature to cope with unbounded domains. Among these methods there is the Perfectly Matched Layer (PML) method. PML is based on simulating an absorbing layer surrounding the domain of interest, like a thin sponge which absorbs the scattered field radiated to the exterior of this domain. This method is known as "perfectly matched" because the interface between the physical domain of interest and the absorbing layer does not produce spurious reflections (in theory and for a continuous problem). In Refs. 15 and 16, we proposed a PML for aeroacoustic problems using Galbrun equation in the presence of an axial and a swirling steady mean flow in a system of cylindrical coordinates.

\section{Axisymmetric formulation}

An axisymmetric duct geometry sketched on the $(r, \theta, z)$ cutting plane is now considered in a cylindrical coordinate system with axisymmetric mean flow as shown in Fig. 3. All perturbations are harmonics with an $e^{-\mathrm{i} \omega t}$ time dependence and a generic acoustic field is given by

$$
\left(\mathbf{w}^{L}, p^{L}\right)(r, \theta, z, t)=\left(\mathbf{w}^{L}, p^{L}\right)(r, z) e^{\mathrm{i}(m \theta-\omega t)},
$$

where $m$ is the azimuthal mode order.

\section{E. Variational formulation of axisymmetric problem}

In order to solve the system Eq. (13) by FEM, the equations are, respectively, multiplied by the trial fields $\mathbf{w}^{*}$ and $p^{*}$, and integrated over the acoustic domain $\Omega$. Integrating by parts and applying the boundary conditions [Eq. (14) and Eq. (15)] yields the following variational problem

$$
\begin{aligned}
& \int_{\Omega}\left(-\frac{1}{\rho_{0} c_{0}^{2}} p^{*} p^{L}+\mathbf{w}^{*} \cdot \nabla p^{L}+\nabla p^{*} \cdot \mathbf{w}^{L}-\omega^{2} \rho_{0} \mathbf{w}^{*} \cdot \mathbf{w}^{L}\right. \\
& \quad-\mathrm{i} \omega \rho_{0} \mathbf{w}^{*} \cdot\left(\mathbf{v}_{\mathbf{0}} \cdot \nabla \mathbf{w}^{L}\right)+\mathrm{i} \omega \rho_{0}\left(\mathbf{v}_{\mathbf{0}} \cdot \nabla \mathbf{w}^{*}\right) \cdot \mathbf{w}^{L}-\rho_{0}\left(\mathbf{v}_{0} \cdot \nabla \mathbf{w}^{*}\right) \cdot\left(\mathbf{v}_{\mathbf{0}} \cdot \nabla \mathbf{w}^{L}\right) \\
& \left.+\mathbf{w}^{*} \cdot \nabla p_{0}\left(\nabla \cdot \mathbf{w}^{L}\right)-\mathbf{w}^{*} \cdot\left({ }^{T} \nabla \mathbf{w}^{L} \cdot \nabla p_{0}\right)\right) r \mathrm{~d} r \mathrm{~d} \theta \mathrm{d} z \\
& \quad+\int_{S_{2}}\left(\frac{1}{\mathrm{i} \omega Z} p^{*} p^{L}\right) \mathrm{d} S=\mathbf{0} \quad \forall\left(\mathbf{w}^{*}, p^{*}\right) \quad \text { with } \quad\left(\left.\mathbf{w}^{*}\right|_{S_{1}}=0,\left.p^{*}\right|_{S_{1}}=0\right) .
\end{aligned}
$$

Line 1 represents the no-flow operators. Line 2 represents the mean flow effects. Line 3 results from the variations in the mean pressure $p_{0}$ and the last line is the contribution of the liner boundary surfaces. In PML, the variational formulation is close to the above formulation, where we introduce the absorption coefficient. 


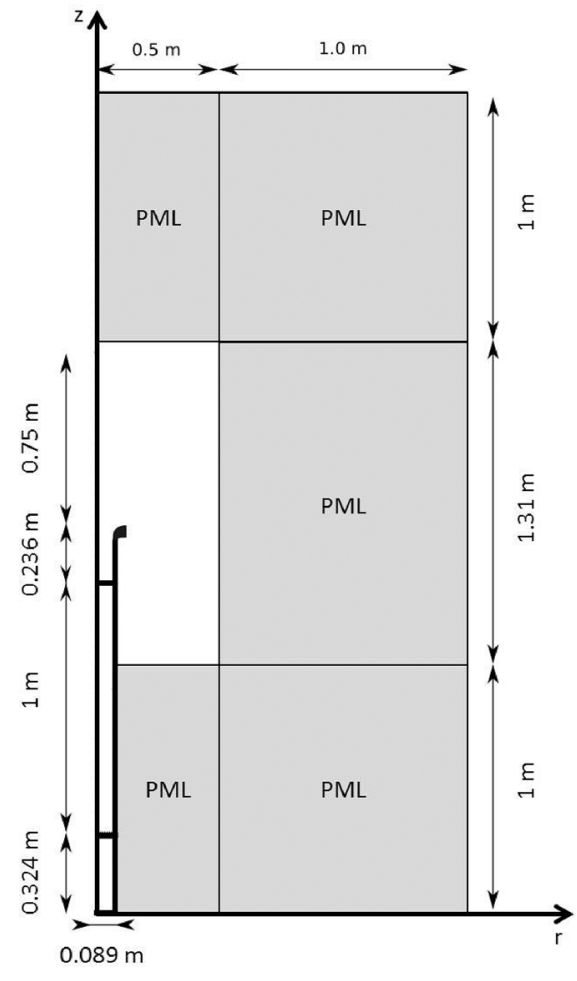

FIG. 3. Numerical model geometry.

\section{F. Finite element discretization}

The total formulation in the whole domain is obtained by simply applying the two variational formulations Eq. (17) and the modified formulation in the PML domain ${ }^{15}$ written in the same geometric coordinates. The standard procedure of finite element discretization is used. The key to the success of the mixed finite element approach is to choose the appropriate interpolation of displacement and pressure that satisfies the inf-sup condition. ${ }^{17}$ The triangular linear element T4-3c with a "bubble" function for the displacement, ${ }^{13}$ which may be referred to as the MINI element in the literature, is the one used in this paper.

After assembling and applying the boundary conditions, the global discretized variational formulation yields the following algebraic system

$$
\mathbf{K}(\omega) \mathbf{U}(\omega)=\mathbf{F}(\omega),
$$

where $\mathbf{U}(\omega)$ contains all the acoustic nodal unknowns (displacement and pressure). The matrix $\mathbf{K}(\omega)$ is unsymmetrical, complex, and band. A sparse storage is chosen. For a fixed $\omega$, the unknown nodal vector $\mathbf{U}(\omega)$ is finally obtained by using an LU decomposition.

\section{EXPERIMENTAL SETUP AND DATA PROCESSING FOR MULTIMODAL PROPAGATION IN TREATED DUCT WITH FLOW}

This section presents the development of an experimental method for ducts with an acoustic treatment. We describe the different components of the experimental device, and we present the techniques for processing the measurement data.

\section{A. Experimental setup}

The experimental measurements were performed on our aeroacoustic test facilities located in the anechoic chamber of the Roberval laboratory. Figure 4 shows a picture of the open ended duct and measurement sections in the anechoic chamber. A schematic illustration of this assembly is shown in Fig. 5, presenting an overview of the experimental device.

The main components of the test facilities are as follows:

- A cylindrical steel duct having a thickness of $0.015 \mathrm{~m}$ and an internal diameter of $0.148 \mathrm{~m}$.

- A centrifugal fan located outside the anechoic chamber for generating a negative or positive mean steady flow up to Mach 0.25 . The axial flow velocity is measured using a Pitot probe in the center of the fan outlet duct section.

- A sound generation system formed by pressure chambers, with sensitivity (sound pressure obtained at $1 \mathrm{~m}$ where $1 \mathrm{~W}$ is applied) of $118 \mathrm{~dB} / 1 \mathrm{~W} / 1 \mathrm{~m}$, bandwidth of 250 to $9000 \mathrm{~Hz}$ and maximum sound pressure level of $136 \mathrm{~dB}$ at $150 \mathrm{~W}$. During measurement, the source is driven by a multi-sines signal via a power amplifier. The number of sines imposed by the computer is set at 79 , from 256 to $4992 \mathrm{~Hz}$ with steps of $64 \mathrm{~Hz}$. The input signal of the sound source is generated by the computer and is the reference signal of the acquisition chain.

- A removable test section wherein an acoustic treatment of $0.3 \mathrm{~m}$ length can be applied.

- Two measurements sections on either side of the removable section: $S_{1}$ before treatment and $S_{2}$ after treatment (relative to the position of the source).

In each section, a probe performs local pressure measurements using a microphone with noise cone. Each probe moves to 240 points (16 azimuthal positions $\times 15$ radial positions). The displacement of these probes is directly controlled by the computer. The exploration of the probe provides the pressure field in the measurement section and the discretization allows a modal decomposition of the azimuthal modes up to $m= \pm 7$.

The calibration of the microphones is based on transfer functions determined by comparing the microphones' signals with the noise cone to that of a reference microphone. ${ }^{18}$ The validity range of the microphone calibration is from 0 to $5000 \mathrm{~Hz}$.

The acquisition system consists of an analog to digital conversion chain connected to a computer with a data processing program.

- Different rigid bellmouths can be fixed at the end of the duct. We have chosen an exponential termination to minimize the turbulence related to the flow at the end of the duct for an aspiring or blowing air flow.

\section{Signal processing}

The pressure at each microphone $j$ (for $j=[1,2]$ ) is defined here as the component of the signal that is correlated with the excitation signal from the generator ${ }^{19}$ 


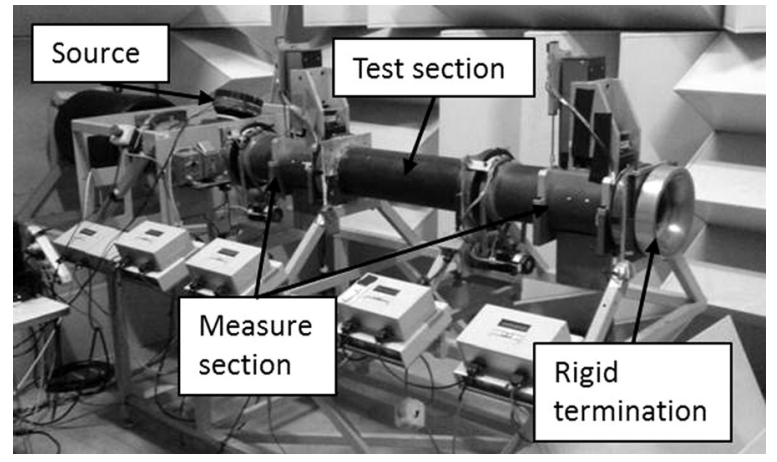

FIG. 4. Picture of the open ended duct in the anechoic chamber.

$$
P_{M_{j}}(r, \theta, f)=\frac{G_{\text {ref }, M_{j}}(r, \theta, f)}{G_{\text {ref, }, \text { ref }}(f)} \cdot \frac{\left|S_{\text {ref }}(f)\right|}{H_{M_{j}}}
$$

with $G_{\mathrm{ref}, M_{j}}(r, \theta, f)=S_{\mathrm{ref}}^{*}(f) \cdot S_{M_{j}}(r, \theta, f)$ the cross spectrum between the reference signal $S_{\text {ref }}(f)$ (signal from the generator) and the microphone signal $S_{M_{j}}(r, \theta, f), G_{\text {ref,ref }}(f)$ $=S_{\text {ref }}^{*}(f) \cdot S_{\text {ref }}(f)$ self-spectrum of the reference signal, $H_{M_{j}}$ the calibration transfer function of the microphone $j$ in $\mathrm{V} / \mathrm{Pa}$ and $\left|S_{\text {ref }}(f)\right|$ the absolute value of the reference signal.

At the end of this signal processing, the user gets the value of the (complex) acoustic pressure in Pa measured at about 240 points of each section for 79 frequencies from 256 to $4992 \mathrm{~Hz}$ in steps of $64 \mathrm{~Hz}$.

\section{Test section}

The treated section is made from a perforated aluminium plate on which are arranged $8 \times 16$ rectangular cavities $(38 \mathrm{~mm} \times 29 \mathrm{~mm})$ which are $8 \mathrm{~mm}$ deep (see Fig. 6). The whole is enclosed by two rigid aluminium cylinders, making a rigid bottom for the cavities. This stack, assumed locally reactive (at least until the cutoff frequency of the cavity to $4500 \mathrm{~Hz}$ ) and axisymmetric, simulates an SDOF treatment. Two test sections are available with different configurations of plates. The first test section has a perforated plate, and the second a microperforated plate whose characteristics are summarized in Table II.

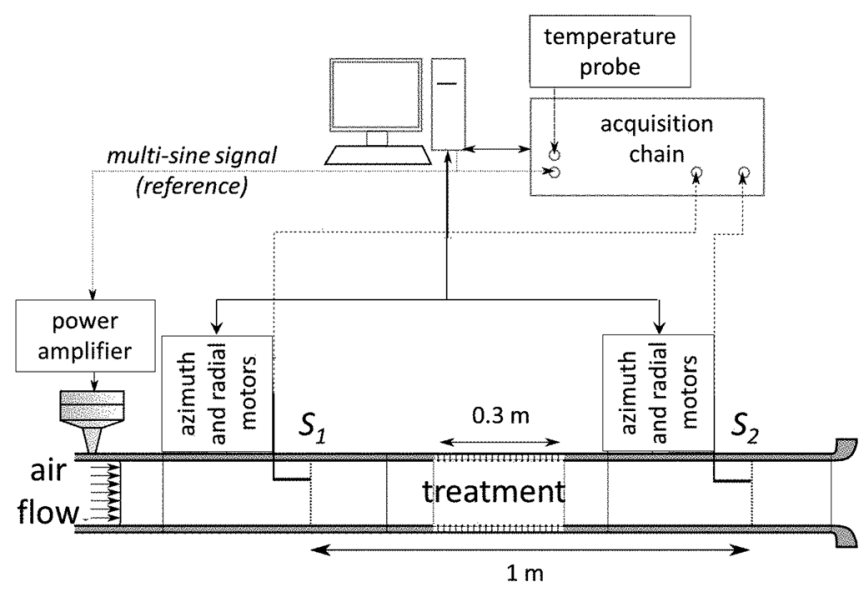

FIG. 5. Sketch of experimental device.

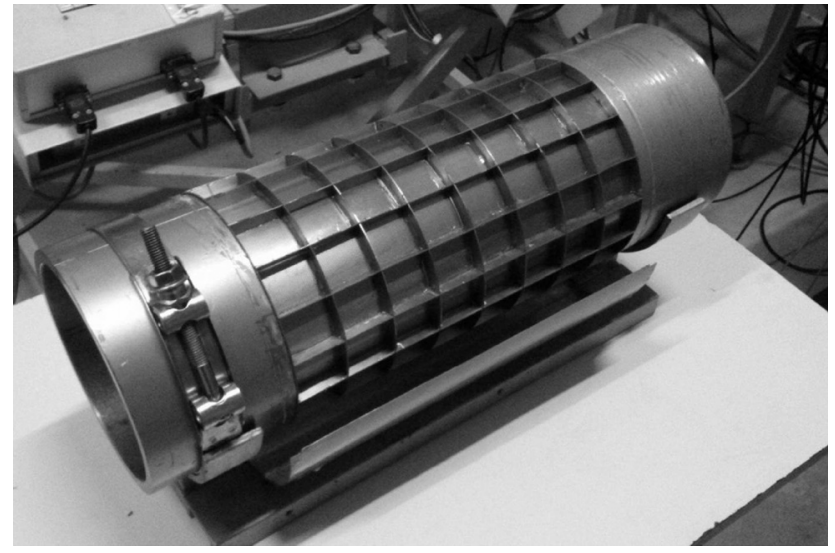

FIG. 6. Test section with microperfored plate and rectangular cavities (without rigid bottom).

\section{The propagation modes in the duct}

We are interested in the propagation in the frequency band $[250-5000 \mathrm{~Hz}]$. The dispersion equation of an infinite duct with circular cross section and with a uniform mean flow is given by

$$
k_{r}^{2}+k_{z}^{2}-\left(k-M k_{z}\right)^{2}=0
$$

With rigid walls, the boundary condition satisfies $J_{m}^{\prime}\left(k_{r} a\right)=0$ where $J_{m}$ is the Bessel function of the first kind of order $m$. This equation can be satisfied only for certain discrete values of $k_{r}$, denoted by $k_{r, m n}$, with $n \in \mathbb{N}$. This defines the axial wave number as

$$
k_{z, m n}^{ \pm}=\frac{-k M \pm \sqrt{k^{2}-\left(1-M^{2}\right) k_{r, m n}^{2}}}{\left(1-M^{2}\right)} .
$$

The frequency at which a mode $(m, n)$ begins to propagate is the cutoff frequency of the mode $f_{c, m n}=\left(c_{0} k_{r, m n} / 2 \pi\right)$ $\sqrt{\left(1-M^{2}\right)}$ for uniform flow. The cutoff frequencies of the first modes are given in Table III.

\section{B. Numerical setup}

The numerical method is based on the mixed finiteelement pressure-displacement formulation based on Galbrun's equation and an axisymmetric PML ${ }^{15}$ (see Sec. III). The geometry of the model reproduces that of the experimental devices. The PML domain simulates the walls of an anechoic chamber. The length of the PML and its absorption parameters were optimized to eliminate reflections over the frequency band. Thus, $L_{p m l}$ is set at $1.0 \mathrm{~m}$ and the absorption parameter $\beta$ at 4000 in each direction. In this configuration, the PML offers good performance even at low frequencies and near the cutoff frequencies.

TABLE II. Plate parameters of tested SDOF treatments.

\begin{tabular}{lcc}
\hline \hline Plate type & Perforated & Microperforated \\
\hline Plate thickness $t(\mathrm{~mm})$ & 0.5 & 0.5 \\
Hole diameter $d(\mathrm{~mm})$ & 1.89 & 0.30 \\
Perforation rate $\sigma$ & 0.0176 & 0.0116 \\
\hline
\end{tabular}


TABLE III. Analytical cutoff frequencies $(\mathrm{Hz})$ of the modes in our circular duct with an uniform flow.

\begin{tabular}{lcccccccc}
\hline \hline$(\mathrm{m}, \mathrm{n})$ & $( \pm 1,0)$ & $( \pm 2,0)$ & $(0,1)$ & $( \pm 3,0)$ & $( \pm 4,0)$ & $( \pm 1,1)$ & $( \pm 5,0)$ & $( \pm 2,1)$ \\
\hline$M_{0}=0$ & 1357 & 2251 & 2824 & 3097 & 3920 & 3930 & 4729 & 4943 \\
$M_{0}=0.25$ & 1322 & 2192 & 2750 & 3016 & 3817 & 3827 & 4605 & 4814 \\
\hline \hline
\end{tabular}

The input section of the duct, taken as a sound source, corresponds to the measurement section $S_{1}$ of the experimental data. The output section, corresponding to measurement section $S_{2}$, is used for comparison of the numerical and experimental results.

The computational domain has three different mesh refinements to ensure accurate results while minimizing the size of the problem. The duct interior has a fine mesh size of $\lambda_{5000 \mathrm{~Hz}} / 12$, the area near the termination a mesh size of $\lambda_{5000 \mathrm{~Hz}} / 5$ and the PML a mesh size of $0.05 \mathrm{~m}$ at the ends of the field. Employing a PML with a progressively increasing mesh does not degrade the accuracy of the results. The entire model has 14594 nodes and 30520 elements.

The flow is calculated numerically using the CFD Fluent software. This method allows the description of a realistic flow into the duct and at the termination, thus overcoming the lack of experimental measurements of the flow profile in the pipe. For this, an incompressible turbulent model $k$-epsilon, respecting a condition of no-slip at the wall, is applied. The results obtained under Fluent are then fed into the numerical code to each node of the mesh. For flow at $M_{0}= \pm 0.25$, the flow profile in section $S_{2}$ is presented in Fig. 7.

\section{Protocol for comparison of numerical and experimental results}

The first step is to obtain, after signal processing (see Sec. IV A 1), the experimental acoustic pressure field measured in section $S_{1}$ for different frequencies and at 240 measurement points. Then, an azimuthal modal decomposition is performed using the spatial Fourier transform to obtain the radial profiles necessary for the input data of the software axisymmetric code. Indeed, the pressure field inside the duct may be written

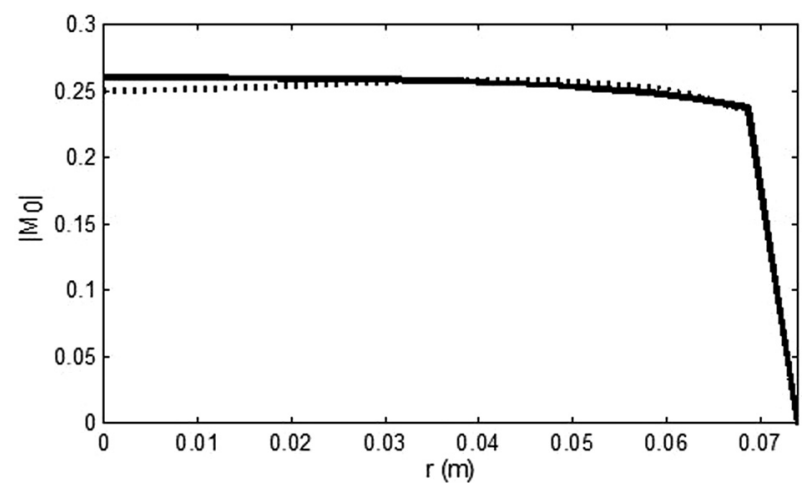

FIG. 7. Flow velocity profile (Mach number) calculated by Fluent at the measuring section $S_{2}$ for $M_{0}=+0.25(-)$ and $M_{0}=-0.25(\cdots)$.

$$
p(r, \theta, z, f)=\sum_{m=-\infty}^{+\infty} p_{m}(r, z, f) e^{i m \theta}
$$

In section $S_{1}$ at a given frequency $f$ for the $m$ th azimuth mode, the pressure can be expressed by

$$
p_{m}\left(r, z_{1}, f\right)=\frac{1}{2 \pi}\left\langle p\left(r, \theta, z_{1}, f\right) \mid e^{i m \theta}\right\rangle
$$

with $\langle\cdot \mid \cdot\rangle$ the scalar product. This decomposition is made for $-7 \leq m \leq+7$.

These pressure profiles (for each mode and each frequency) are then imposed on the nodes of section $S_{1}$ as input for the numerical code. For each calculation, we extract the radial profile in section $S_{2}$ as a result. The total pressure field is then reconstructed for section $S_{2}$ using the inverse of the Fourier transform

$$
p\left(r, \theta, z_{2}, f\right)=\sum_{m=-7}^{+7} p_{m}\left(r, z_{2}, f\right) e^{-i m \theta} .
$$

The comparison of experimental and numerical results in the duct is achieved by comparing the difference of total energy level of acoustic pressure between section $S_{1}$ and section $S_{2}$

$$
\Delta L(f)=L\left(z_{2}, f\right)-L\left(z_{1}, f\right)
$$

with energy level of acoustic pressure p $^{20}$

$$
\begin{aligned}
& L(z, f)=10 \log \left(\frac{E_{T}^{p}(z, f)}{\pi R^{2}\left(2 \times 10^{-5}\right)^{2}}\right) \text { in } \mathrm{dB} \\
& E_{T}^{p}(z, f)=\int_{0}^{2 \pi} \int_{0}^{R}|p(r, \theta, z, f)|^{2} r \mathrm{~d} r \mathrm{~d} \theta
\end{aligned}
$$

and $R$ the inner radius of the duct.

\section{Validation of the approach with a rigid test section}

To validate the entire process, we first applied it to the case of a rigid tube (without treatment) without flow, and with a positive and negative flow $\left(M_{0}= \pm 0.25\right)$. The level of sound pressure in section $S_{1}$ is around $105 \mathrm{~dB}$ like all presented results.

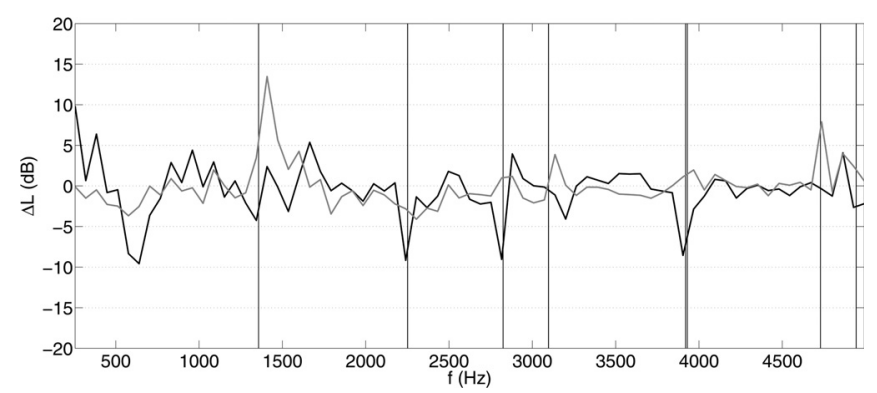

FIG. 8. Difference of the total energy level of the sound pressure between $S_{1}$ and $S_{2}$ for rigid untreated test section without flow: experimental results (gray), numerical results (black). 


\section{Without flow}

Figure 8 presents the curves of the following:

- The experimental difference of total energy level between section $S_{1}$ and section $S_{2}$.

- The numerical difference of total energy level between section $S_{1}$ and section $S_{2}$.

The vertical lines indicate the acoustic modes cutoff frequencies (see Table III).

Figure 8 shows the very good agreement between the numerical and experimental difference of energy levels outside the cutoff frequencies, where the change in the nature of the behaviour requires high precision. Inaccuracies in the numerical geometry, imperfection in the experimental duct, wall vibration phenomena and acoustic leakage (not taken into account by the numerical model) may explain the approximately $3 \mathrm{~dB}$ difference. Furthermore, we checked for the conservation of energy between the two measurement sections.

\section{With flow}

Figure 9 shows the numerical and experimental results with a positive $M_{0}=+0.25$ and negative $M_{0}=-0.25$ axial mean steady flow. In both cases, the results are satisfactory, with differences of approximately $3 \mathrm{~dB}$ like without mean flow, and verify the energy conservation over a wide frequency band.

\section{RESULTS}

The method developed above was used to test SDOF treatment impedance models with multimodal propagation in mean steady flow. Two SDOF treatments were tested, one of which was provided with a perforated plate, and the other with a microperforated plate (see Table II). Several flow
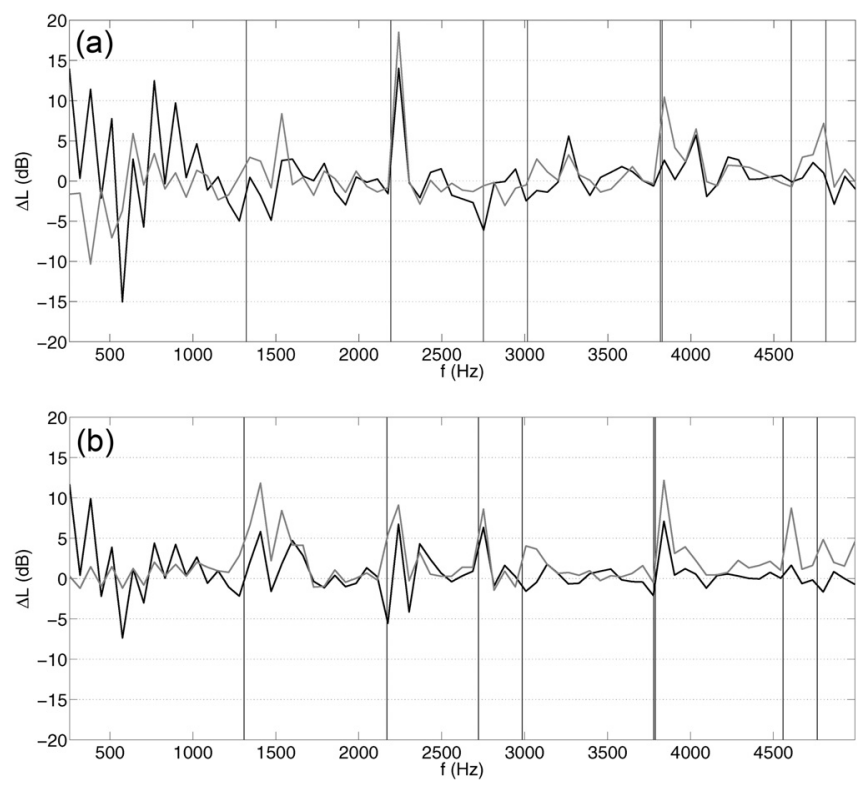

FIG. 9. Difference of the total energy level of the sound pressure between $S_{1}$ and $S_{2}$ for rigid untreated test section with flow at $M_{0}=+0.25$ (a) and $M_{0}=-0.25$ (b): experimental results (gray), numerical results (black). rates were applied for $-0.25 \leq M_{0} \leq 0.25$. The impedance models were introduced into the numerical code, as boundary conditions in the treated part [see Eq. (15) and Eq. (17)], and the numerical results were compared with the measurements. We recall that for the following, the comparisons are valid in the frequency range 250 to $4500 \mathrm{~Hz}$ (see the remarks on the source bandwidth and the assumption that the cavity is locally reactive).

\section{A. SDOF treatment with perforated plate}

Among all the presented acoustic impedance models, those of $\mathrm{Yu}$ and Guess are adapted to the perforated plates $(d>t)$.

\section{Without flow}

Figure 10 shows an agreement between numerical and experimental difference of total energy levels inside the range of effectiveness of the treatment, regardless of the impedance model.

The tuning frequency is close to $1600 \mathrm{~Hz}$, depending on the model. For this tuning frequency, the pressures are very low in section $S_{2}$ and so is difficult to have significant experimental pressure due to background noise. The slightest deviation is amplified by the decibel representation.

The quality of the results attests to the validity of the impedance models without flow, despite the perforation rate differences between the treatment employed and those used by the authors (see Tables I and II). The numerical results with Yu's model better agree with the experimental results for this configuration, with a difference of less than approximately $2 \mathrm{~dB}$.

It is interesting to note that although most of the acoustic impedance models were validated in plane wave by their authors, they remain valid up to now in multimodal. ${ }^{21}$

\section{With positive flow}

Figures 11 give the results for $M_{0}=+0.1$ and $M_{0}=+0.25$. For $M=0.1$, the tuning frequency is close to $1700 \mathrm{~Hz}$, whereas for $M=0.25$ close to $3000 \mathrm{~Hz}$. For $M=0.25$, the treatment efficiency is less pronounced and is spread out over a wide band of frequencies following the model. A better result is observed for $M=0.25(<2 \mathrm{~dB}$ for $f<3800 \mathrm{~Hz}$ like without flow) than for $M=0.1$. The

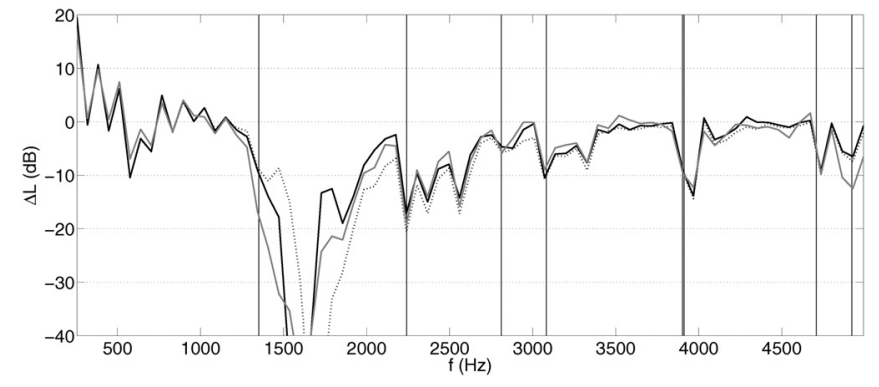

FIG. 10. Difference of the total energy level of the sound pressure between $S_{1}$ and $S_{2}$ for SDOF treatment with perforated plate test section without flow: experimental results (gray -), numerical results with the model of $\mathrm{Yu}$ (black-) and Guess (black …). 


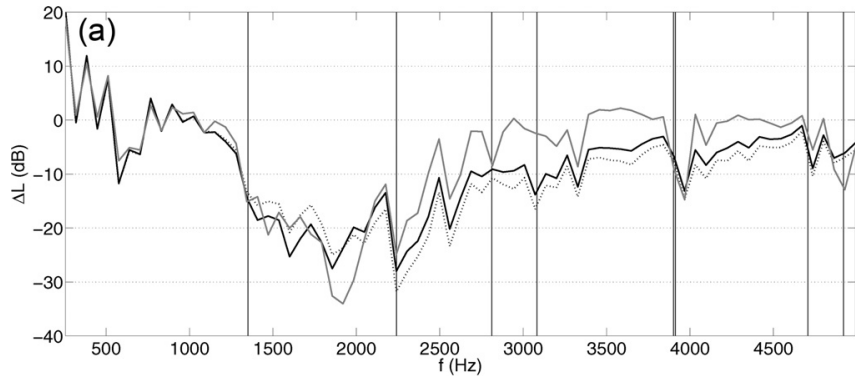

(b)

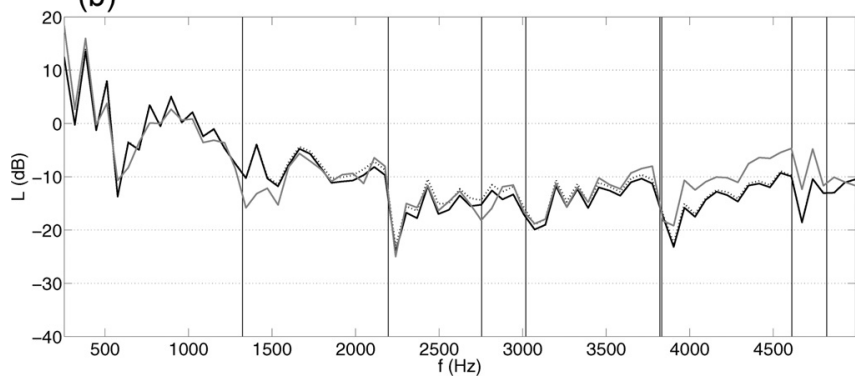

FIG. 11. Difference of the total energy level of the sound pressure between $S_{1}$ and $S_{2}$ for SDOF treatment with perforated plate test section with flow at $M_{0}=+0.10$ (a) and $M_{0}=+0.25$ (b): experimental results (gray -); numerical results with the model of $\mathrm{Yu}$ (black -) and Guess (black ...).

differences could be due to the fact that the applied flow rate is not high enough compared to the validation range used by the authors of those models (see Table I).

The numerical simulations were carried out in the presence of shear flow (from a calculation using Fluent) with a boundary layer thickness of $\delta_{e}=5 \mathrm{~mm}$, that is around $7 \%$ of the duct radius (see Fig. 7). To determine the influence of the aerodynamic shear boundary layer associated with a non-slip condition (zero speed to the wall), the same calculations were performed using a flow resulting from the resolution of the Laplace equation. This is a potential flow ensuring a nonsheared profile in the duct. The results are shown in Fig. 12, only for the Guess model. The absorption treatment is lightly amplified with the presence of the boundary layer. Indeed, when the shear flow propagates in the direction of the acoustic wave, it refracts the wave on the treated wall, which increases its attenuation. The effect of shear and the no-slip condition is however negligible in the case of a positive flow (a $1 \mathrm{~dB}$ difference between the two calculations).

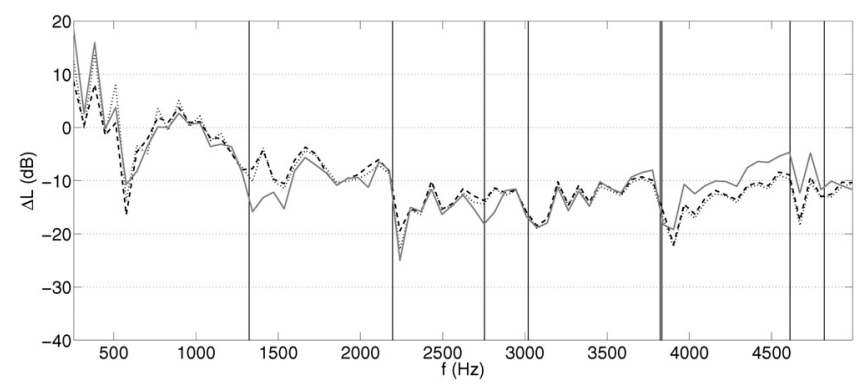

FIG. 12. Difference of the total energy level of the sound pressure between $S_{1}$ and $S_{2}$ for SDOF treatment with perforated plate test section with flow at $M_{0}=+0.25$ : experimental results (gray -); numerical results with the model of Guess with shear flow (black ...) and potential flow (black --)

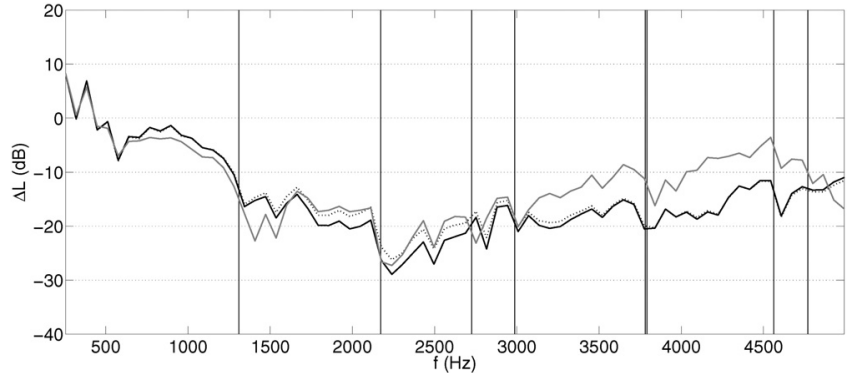

FIG. 13. Difference of the total energy level of the sound pressure between $S_{1}$ and $S_{2}$ for SDOF treatment with perforated plate test section with flow at $M_{0}=-0.25$ : experimental results (gray - ); numerical results with the model of $\mathrm{Yu}($ black -) and Guess (black $\cdots)$.

\section{With negative flow}

None of the acoustic impedance models studied specified the direction of flow relative to that of the acoustic wave in their formulation ( $M_{0}$ is considered positive). The relative direction of flow should not affect the impedance of the liner, as shown by Renou ${ }^{22}$ and Watson. ${ }^{23}$

Figure 13 shows the results for $M_{0}=-0.25$. Below $f<3000 \mathrm{~Hz}$, the comparison is satisfactory for all models $(<2 \mathrm{~dB}$ for Yu model).

The flow assuming a sheared flow has a boundary layer thickness of $\delta_{e}=5 \mathrm{~mm}$. Figure 14 compares the previous result for the Guess model to those calculated with a potential flow. The boundary layer visibly reduces the liner absorption. Indeed, when a shear flow propagates against the direction of the acoustic wave, it refracts the wave inside the duct, which limits its attenuation. Unlike the case of a positive flow, these effects are not negligible, with differences of up to approximately $3 \mathrm{~dB}$ between the two calculations. Finally, for the case of a negative flow, the quality of the simulation is much more sensitive to the realism of the flow in the tube that in the positive case. Thus, a boundary layer larger may be more representative of the real boundary layer and could improve the numerical simulation results.

\section{B. SDOF treatment with microperforated plate}

In this section, the acoustic impedance models adapted to the microperforated plates $(d<t)$, namely, those of Yu, Cobo and Allam (2008 and 2011), are tested. Only the configurations without flow and positive flow are described below.

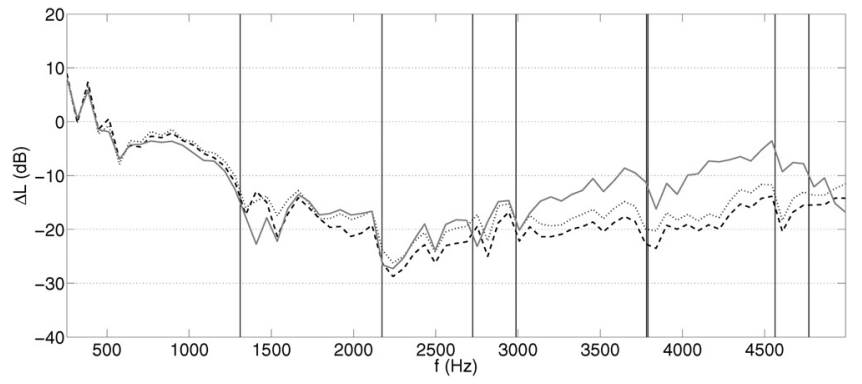

FIG. 14. Difference of the total energy level of the sound pressure between $S_{1}$ and $S_{2}$ for SDOF treatment with perforated plate test section with flow at $M_{0}=-0.25$ : experimental results (gray - ); numerical results with the model of Guess with shear flow (black $\cdots$ ) and potential flow (black --). 


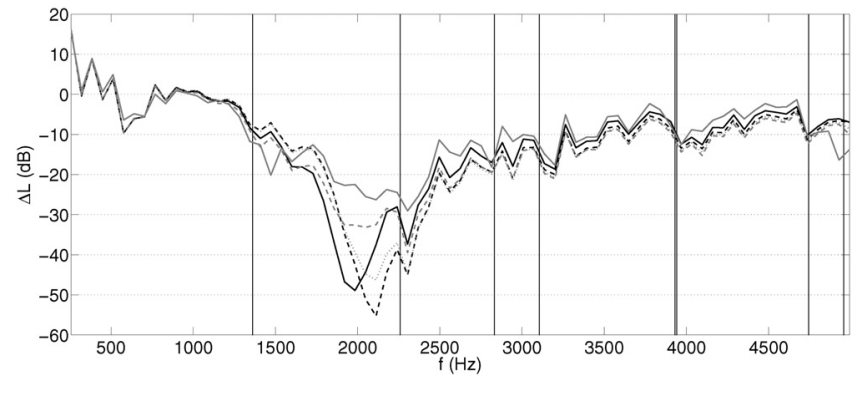

FIG. 15. Difference of the total energy level of the sound pressure between $S_{1}$ and $S_{2}$ for SDOF treatment with microperforated plate test section without flow: experimental results on the section $S_{2}$ (gray -); numerical results with the model of Yu (black -); Cobo (black - -); Allam 2011 (gray - -); Allam 2008 (gray …).

\section{Without flow}

Figure 15 shows the results without mean flow. As for the perforated plate, the results agree well for all models except for an area close to the tuning frequency for the same reasons explained above. Here again we find the tuning frequency provided around $2200 \mathrm{~Hz}$ depending on the models. The Yu model seems a bit more efficient than the others.

\section{With positive flow}

Figure 16 gives the results with mean flow at $M_{0}=$ +0.1 and $M_{0}=+0.25$. For $M=0.1$, the tuning frequency is close to $2300 \mathrm{~Hz}$, whereas for $M=0.25$ is close to $3000 \mathrm{~Hz}$. As for the perforated plate, for $M=0.25$, the treatment efficiency is less pronounced and is spread out over a wide band of frequencies following the model. A better result is observed for $M=0.25$ than for $M=0.1$. We attribute this to the last reasons given above for perforated plate. As with the perforated processing, the $\mathrm{Yu}$ model is better $(<2 \mathrm{~dB})$ suited to the high-frequency experimental results.
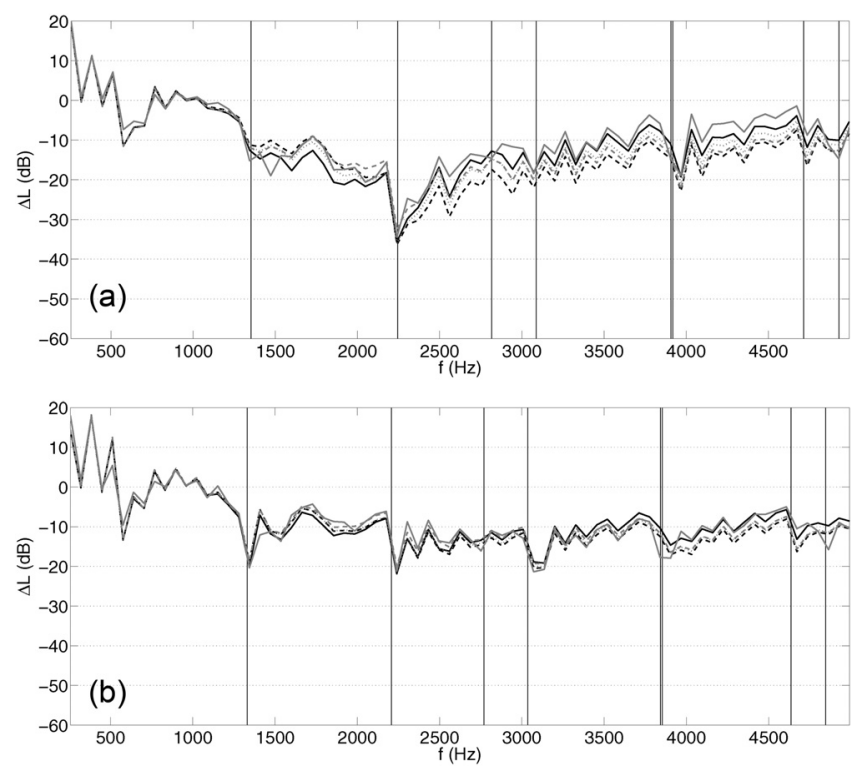

FIG. 16. Difference of the total energy level of the sound pressure between $S_{1}$ and $S_{2}$ for SDOF treatment with microperforated plate test section with flow at $M_{0}=+0.10$ (a) and $M_{0}=+0.25$ (b): experimental results (gray -); numerical results with the model of Yu (black -); Cobo (black --); Allam 2011 (gray --); Allam 2008 (gray - .).
This second series of tests with microperforated plate confirms the general conclusions of the first with perforated plate.

\section{CONCLUSION}

An experimental/numerical method for ducts with acoustic treatment was presented. The validation of the method was made on a rigid section with differences of $<3 \mathrm{~dB}$ between the numerical and experimental results. This method is relatively robust, simple and efficient for testing impedance models of acoustic treatments. It can be used subsequently to improve the impedance models in the presence of more complex mean flows and under multimodal excitation.

Several acoustic impedance models taking into account the flow were then tested in multimodal condition on two types of SDOF treatment: perforated and microperforated plate. Regarding the SDOF treatment with perforated plate without flow, the results obtained with the models of $\mathrm{Yu}$ and Guess are in good agreement with experimental measurements with the hypothesis of a localized reaction. With positive flow $\left(M_{0}=+0.1\right.$ and $\left.M_{0}=+0.25\right)$, gaps emerge but decrease as the flow rate increases. This difference is attributed to the fact that the flow rate is lower than those used by the authors of the models for validation. With numerical simulations, the effects of the aerodynamic boundary layer shear flow are shown relatively unimportant in the case of a positive flow. For negative flow $\left(M_{0}=-0.25\right)$, the results are very dependent on the nature of the flow (shear size of the boundary layer, slip condition at the wall). In addition, both models are very similar.

For an SDOF treatment with microperforated plate without flow, numerical results with the model of Yu, Cobo and Allam (2008 and 2011) approximate the experimental results, and especially those of Yu. Similarly, in the presence of a positive flow $\left(M_{0}=+0.25\right)$, the simulations agree well with experiment for all models.

$\mathrm{Yu}$ model is the only one tested for perforated or microperforated plate. It gives the numerical results with approximately $2 \mathrm{~dB}$ difference with the experimental results for perforated or microperforated plate and without flow or with flow at $M_{0}=0.25$ despite the perforation rate differences between the treatment employed and those used by Yu.

TABLE IV. Effects taken into account by the authors.

\begin{tabular}{|c|c|c|c|c|c|}
\hline & Guess $^{2}$ & $\begin{array}{l}\text { Allam } \\
\text { and } \\
\text { Abom }^{3}\end{array}$ & $\begin{array}{c}\mathrm{Yu} \\
\text { et al. }\end{array}$ & $\begin{array}{l}\text { Cobo } \\
\text { et al. }\end{array}$ & $\begin{array}{l}\text { Allam } \\
\text { and } \\
\text { Åbom }{ }^{7}\end{array}$ \\
\hline \multicolumn{6}{|l|}{ Resistance } \\
\hline Effects of viscosity & $\mathrm{x}$ & $\mathrm{x}$ & $\mathrm{x}$ & $\mathrm{x}$ & $\mathrm{x}$ \\
\hline Effects of radiation & $\mathrm{x}$ & & & & $\mathrm{x}$ \\
\hline Effects of acoustic velocity & $\mathrm{x}$ & & $\mathrm{x}$ & & $\mathrm{x}$ \\
\hline Effects of mean flow & $\mathrm{x}$ & $\mathrm{x}$ & $\mathrm{x}$ & $\mathrm{x}$ & $\mathrm{x}$ \\
\hline \multicolumn{6}{|l|}{ Reactance } \\
\hline Effects of viscosity & $\mathrm{x}$ & $\mathrm{x}$ & $\mathrm{x}$ & $\mathrm{x}$ & $\mathrm{x}$ \\
\hline Effects of radiation & $\mathrm{x}$ & $\mathrm{x}$ & & $\mathrm{x}$ & $\mathrm{x}$ \\
\hline Effects of acoustic velocity & $\mathrm{x}$ & & $\mathrm{x}$ & & $\mathrm{x}$ \\
\hline Effects of mean flow & $\mathrm{x}$ & $\mathrm{x}$ & & $\mathrm{x}$ & $\mathrm{x}$ \\
\hline $\begin{array}{l}\text { Interaction effects between } \\
\text { adjacent holes }\end{array}$ & $\mathrm{x}$ & $\mathrm{x}$ & & $\mathrm{x}$ & \\
\hline
\end{tabular}




\section{APPENDIX}

Table IV gives a summary of the effects taken into account by the authors.

${ }^{1}$ A. H. Marsh, "Study of acoustical treatments for jet-engine nacelles," J. Acoust. Soc. Am. 43, 1137-1156 (1968).

${ }^{2} \mathrm{~A}$. W. Guess, "Calculation of perforated plate liner parameters from specified acoustic resistance and reactance," J. Sound Vib. 40(1), 119-137 (1975).

${ }^{3}$ S. Allam and M. Åbom, "Experimental characterization of acoustic liners with extended reaction," in 14th AIAA/CEAS Aeroacoustics Conference (2008).

${ }^{4}$ A. B. Bauer, "Impedance theory and measurements on porous acoustic liners," J. Aircraft 14(8), 720-728 (1977).

${ }^{5}$ J. Yu, M. Ruiz, and H. W. Kwan, "Validation of Goodrich perforate liner impedance model using NASA Langley test data," in 14th AIAA/CEAS Aeroacoustics Conference (29th AIAA Aeroacoustics Conference), Vancouver, British Columbia, Canada (May 5-7, 2008).

${ }^{6} \mathrm{P}$. Cobo, H. Ruiz, and J. Alvarez, "Double-layer microperforated panel/ porous absorber as liner for anechoic closing of the test section in wind tunnels," Acta Acust. Acust. 96, 914-922 (2010).

${ }^{7}$ S. Allam and M. Åbom, "A new type of muffler based on microperforated tubes," J. Vib. Acoust. 133(3), 031005 (2011).

${ }^{8}$ D. Y. Maa, "Theory and design of micro perforated-panel sound absorbing construction," Sci. Sin. 18, 55-71 (1975).

${ }^{9}$ D. Y. Maa, "Microperforated-panel wideband absorbers," Noise Control Eng. J. 29(3), 77-84 (1987).

${ }^{10}$ D. Y. Maa, "Microperforated panel at high sound intensity," in Proceedings of Internoise, Yokohama, Japan (1994).

${ }^{11}$ D. Y. Maa, "Potential of microperforated panel absorber," J. Acoust. Soc. Am. 104(5), 2861-2866 (1998).
${ }^{12}$ R. J. Astley, R. Sugimoto, and P. Mustafi, "Computational aero-acoustics for fan duct propagation and radiation.Current status and application to turbofan liner optimisation,” J. Sound Vib. 330, 3832-3845 (2011).

${ }^{13} \mathrm{~F}$. Treysséde, G. Gabard, and M. B. Tahar, "A mixed finite element method for acoustic wave propagation in moving fluids based on an EulerianLagrangian description," J. Acoust. Soc. Am. 113, 705-716 (2003).

${ }^{14}$ G. Gabard, F. Treysséde, and M. B. Tahar, "A numerical method for vibro-acoustic problems with sheared mean flows," J. Sound Vib. 272, 991-1011 (2004)

${ }^{15}$ R. Baccouche, M. Ben Tahar, and S. Moreau, "Perfectly matched layer for Galbrun's aeroacoustic equation in a cylindrical coordinates system with an axial and a swirling steady mean flow," J. Sound Vib. 378, 124-143 (2016).

${ }^{16} \mathrm{X}$. Feng, M. Ben Tahar, and R. Baccouche, "The aero-acoustic Galbrun equation in the time domain with perfectly matched layer boundary conditions," J. Acoust. Soc. Am. 139(1), 320-331 (2016).

${ }^{17}$ G. Gabard, R. J. Astley, and M. B. Tahar, "Stability and accuracy of finite element methods for flow acoustics. II: Two-dimensional effects," Int. J. Numer. Meth. Eng. 63, 947-973 (2005).

${ }^{18}$ Z. Lazreq and J. M. Ville, "Acoustic calibration of a pressure-velocity probe,” J. Acoust. Soc. Am. 100(1), 364-371 (1996).

${ }^{19}$ J. Y. Chung, "Rejection of flow noise using a coherence function method," J. Acoust. Soc. Am. 62(2), 388-395 (1977).

${ }^{20}$ J. M. Ville and F. Foucart, "Experimental setup for measurement of acoustic power dissipation in lined ducts for higher order modes propagation with air mean-flow conditions," J. Acoust. Soc. Am. 114(4), 1742-1748 (2003).

${ }^{21}$ M. Taktak, J. M. Ville, M. Haddar, G. Gabardl, and F. Foucart, "An indirect method for the characterization of locally reacting liners," J. Acoust. Soc. Am 127(6), 3548-3559 (2010).

${ }^{22}$ Y. Renou and Y. Aurégan, "Failure of the Ingard-Myers boundary condition for a lined duct: An experimental investigation,” J. Acoust. Soc. Am. 130(1), 52-60 (2011).

${ }^{23}$ W. R. Watson and M. G. Jones, "Evaluation of wall boundary conditions for impedance reduction using a dual-source method," AIAA J. 2199, $1-17$ (2012) 